\title{
Potential impact of Helicobacter pylori-related Galectin-3 on chronic kidney, cardiovascular and brain disorders in decompensated cirrhosis
}

Boziki, Marina ; Grigoriadis, Nikolaos ; Doulberis, Michael ; Papaefthymiou, Apostolis ; Polyzos, Stergios A ; Kountouras, Jannis

DOI: https://doi.org/10.1016/j.dld.2019.09.001

Posted at the Zurich Open Repository and Archive, University of Zurich ZORA URL: https://doi.org/10.5167/uzh-187556

Journal Article

Accepted Version

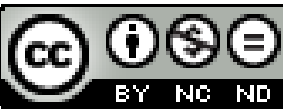

The following work is licensed under a Creative Commons: Attribution-NonCommercial-NoDerivatives 4.0 International (CC BY-NC-ND 4.0) License.

Originally published at:

Boziki, Marina; Grigoriadis, Nikolaos; Doulberis, Michael; Papaefthymiou, Apostolis; Polyzos, Stergios A; Kountouras, Jannis (2020). Potential impact of Helicobacter pylori-related Galectin-3 on chronic kidney, cardiovascular and brain disorders in decompensated cirrhosis. Digestive and Liver Disease, 52(1):121-123.

DOI: https://doi.org/10.1016/j.dld.2019.09.001 


\section{Potential impact of Helicobacter pylori-related Galectin-3 on chronic kidney, cardiovascular and brain disorders in decompensated cirrhosis}

Marina Boziki ${ }^{a}$ MD, PhD, Nikolaos Grigoriadis ${ }^{a}$ MD, PhD, Michael Doulberis ${ }^{b, e}$ MD, DVM, PhD, Apostolis Papaefthymiou $^{c, e}$ MD, Stergios A Polyzos ${ }^{\mathrm{d}, \mathrm{e}}$ MD, Msc, PhD, Jannis Kountouras ${ }^{e}$ MD, PhD

aSecond Neurological Department, Aristotle University of Thessaloniki, AHEPA University General Hospital of Thessaloniki, Thessaloniki 54636, Macedonia, Greece

${ }^{\text {b}}$ Department of Gastroenterology and Hepatology, University of Zurich, Zurich 8091,Switzerland

'Department of Gastroenterology, 401 General Military Hospital of Athens, Athens 11525, Greece

${ }^{\mathrm{d} F i r s t ~ D e p a r t m e n t ~ o f ~ P h a r m a c o l o g y, ~ M e d i c a l ~ S c h o o l, ~ A r i s t o t l e ~ U n i v e r s i t y ~ o f ~}$ Thessaloniki, Thessaloniki 54124, Macedonia, Greece

eDepartment of Internal Medicine, Second Medical Clinic, Ippokration Hospital, Aristotle University of Thessaloniki, Thessaloniki 546 42, Macedonia, Greece 
Jannis Kountouras, MD, PhD

Professor of Medicine

Gastroenterologist

8 Fanariou St, Byzantio

551 33, Thessaloniki, Macedonia, Greece

Tel: +30-2310-892238, Fax: +30-2310-992794

E-mail: jannis@auth.gr, ancoratus2010@gmail.com

Sources of funding / grant support: none. Financial Disclosure: none

Word count: 1500

Keywords: Helicobacter pylori; galectin-3; vascular; renal; multiple sclerosis; dementia; neurodegeneration

Sir,

Oikonomou et al. [1] concluded that Galectin-3 (Gal-3) is a novel marker of chronic kidney disease with predictive ability in decompensated cirrhosis and an index of disease outcome. Notably, although the relationship between plasma concentrations of Gal-3 and estimated glomerular filtration rate (eGFR) is well established [2], a similar association in a cohort of patients with liver cirrhosis has not been studied so far. In this regard, Helicobacter pylori 
infection (Hp-I) is linked with Gal-3 overexpression [3] and appears to be a common denomina- tor of liver cirrhosis, in the context of viral hepatitis, nonalcoholic fatty liver disease (NAFLD), also studied by the authors, and renal insufficiency. For instance, as in the case of NAFLD, $\mathrm{Hp}-\mathrm{I}$ is highly prevalent globally [its current prevalence is 58\% (39.9\%-84.2\%) with increasing tendency due to the effect of immigration] and we postulated that the $\mathrm{Hp}-\mathrm{I}$ might contribute to pathogenesis of NALFD, whereas its eradication may attenuate NAFLD development and/or progression towards advanced liver disease [4]. Moreover, $\mathrm{Hp}-\mathrm{I}$ is strongly associated with hepatitis B- and Crelated cirrho- sis in Europe and in other ethnic populations and $\mathrm{Hp}$ eradication is advocated as a promising approach for the long-term over- all health improvement in patients with chronic viral infection [5]. With respect to $\mathrm{Hp}-\mathrm{I}$ in patients with chronic renal disease, although literature appears conflicting, recent studies demon- strate a connection between $\mathrm{Hp}-\mathrm{I}$ and endstage renal disease [6]. Existing evidence so far underlines the role of increased serum Gal-3 as an indicator of renal function in patients with heart failure. In this respect, Hp-mediated inflammation has been linked with atherosclerosis and the early events of coronary vascular syndrome, thereby influencing the development and/or the progression of cerebro-cardiovascular disease (C-CVD) and suggesting eradication therapy for prevention. Hp eradication might display a positive impact on Hp-related chronic viral infection associated with NAFLD and CVD development/progression [5]. Moreover, there is growing evidence indicating a connection between $\mathrm{Hp}-\mathrm{I}$ and insulin resis- tance (IR) syndrome or metabolic syndrome (MetS) and its related morbidity, including NAFLD and C-CVD, the latter being the end- 
points of MetS [7]. In our series we demonstrated that increased fibrinogen lev- els (an independent risk factor for CVD) are associated with $\mathrm{Hp}-\mathrm{I}$ and can be significantly reduced by $\mathrm{Hp}$ eradication. Several mech- anisms mediated by Hp may be implicated in the perceived risk of CVD. For instance, beyond its role in IR or in increasing fibrino- gen, $\mathrm{Hp}$-I may promote coagulation by stimulating mononuclear cells towards the production of a tissue factor-like procoagulant activity that converts fibrinogen into fibrin; and promoting platelet aggregation via the binding of von Willebrand factor. Likewise, Hp-I may promote the formation of L- and P-selectin-dependent platelet-leukocyte aggregates. Moreover, $\mathrm{Hp}-\mathrm{I}$ induces the expres- sion of plasminogen activator inhibitor-1, as well as increased plasma levels of triglycerides and various atherosclerotic risk fac- tors, such as homocysteine, involved in the pathogenesis of C-CVD $[5,7]$. Other pro-thrombotic mediators and mediators of atheroscle- rosis elevated in the context of $\mathrm{Hp}$-I include the proinflammatory cytokines tumor necrosis factor (TNF)- $\square$ and interleukin (IL)-6, anticardiolipin antibodies and lipid peroxides, also associated with cardiovascular risk [7]. Likewise, serum Gal-3 is related with MetS parameters and has been proposed as an emerging biomarker of the overall risk of CVD [2], as it has been linked with myocar- dial fibrosis and inflammation; Gal-3 overexpression is responsible for the activation of fibroblasts and macrophages with the subsequent development of fibrosis, scar production and, eventually, of cardiac remodeling [2]. Elevated serum Gal-3 is also associated with a higher risk of all-cause mortality and more specifically with CVD mortality and heart failure; Gal-3 is an important predictor of mortality risk in patients with CVD, following adjustment for age and sex; and Gal-3 may also serve as a biomarker for the 
severity of CVD and renal dysfunction, as the two last disorders hav- ing been evaluated via measurements of brain natriuretic peptide (BNP) plus its Nterminal part NT-proBNP and eGFR, respectively [2]. Moreover, Gal-3 has been stated to increase in ischemic brain damage [2]. Taken together, evidence suggests a potential role of Hp-related Gal-3 and MetS as mediators implicated in the chronic failure of var- ious organs, such as liver, heart, kidney and brain, and, thus further research is necessary. Regarding the $\mathrm{Hp}$ related Gal-3 involvement in brain disorders, also connected with liver cirrhosis, we recently reviewed the role of Gal-3 in Hp-related neuroinflammation and subsequent neu- rodegeneration [3]. Under conditions of neuroinflammation, Gal-3 mediates immune cell chemotactic recruitment in the central ner- vous system (CNS), a procedure also triggered by microorganisms, especially in the context of autoimmunity. Experimental evidence stemming from multiple sclerosis (MS) models indicates that Gal- 3 may promote inflammatory infiltration in the CNS parenchyma upon disease exacerbation, a response particularly evident in the sub-ventricular zone, a physiological niche of adult neurogenesis [3]. Relative data also provide evidence of implication of Gal-3 sig- naling in models of traumatic brain injury, CNS neonatal hypoxia and prion disease, all conditions characterized by a strong neu- rodegenerative, and, to a lesser degree, inflammatory pathogenetic component. In this regard, an overall effect of Gal-3 in the cellular components of innate immunity may modulate CNS inflamma- tion and subsequent neurodegeneration [3]. Recent data indicate that Gal-3 is associated with increased risk of cognitive dysfunc- tion (CD), a disorder observed in the majority of patients with liver cirrhosis; Gal-3 is connected 
with poor prognosis of ischemic stroke; and Gal-3 induces amyloid- $\square(\mathrm{A} \square)$ oligomerization and $A \square$ toxicity in Alzheimer's disease (AD). Notably, neuropathol- ogy of hepatic encephalopathy (HE) in cirrhosis is principally of astroglial origin, classified as Alzheimer type 2 astrocytosis, and is further characterized by activation of microglia, suggestive of neuroinflammation. Regarding the Gal-3-mediated mechanisms involved in $\mathrm{Hp}$ - related neurodegeneration, it has been described that $\mathrm{Hp}-\mathrm{I}$ is linked with Gal-3 overexpression by macrophages, which results in reduced macrophage phagocytic capacity and persistent $\mathrm{Hp}$ gastric mucosa colonization [3]. Thus, the assumption that Gal-3 overexpression by the cells of the innate immunity contributes to the capacity of $\mathrm{Hp}$ to circumvent the host's defense mechanisms is further supported. In this respect, gastric mucosa Gal-3 overexpression is a critical endogenous event in $\mathrm{Hp}-\mathrm{I}$ that interferes with various intracellular processes, thereby prolonging cell sur- vival, a feature of gastric oncogenesis [2]. Moreover, one could hypothesize that cells or Hp epitopes stemming from the phago- cytosis of the bacterium and subsequent processing, may access CNS via blood-brain-barrier (BBB) disruption, intranasal inocula- tion or the fast retrograde vagal pathways [3]. In patients with Secondary Progressive MS, a disease type with prominent neurodegenerative pathogenetic component, peripheral blood anti-Gal-3 antibodies recognize BBB structures. It is thus speculated that Gal-3 expressed by the BBB may act as an immunological target for autoimmune cells in MS, thus contributing to the patho- genesis of the disease. Moreover, Gal-3-related BBB disruption may provide brain access to other pathogenic mediators, such as $\mathrm{Hp}$ [3]. Hp might be further involved in BBB breakdown by releasing 
several proinflammatory/vasoactive mediators [8], such as TNF- $\square$ and IL-6, or even defensins, particularly those that display a unique distribution at BBB sites [9]. Hp may activate granulocytes and induce defensins release from granulo- cytes; subsequently, defensins, secreted by activated granulocytes, penetrate the BBB and gain access to the brain, thereby pos- sibly contributing to neurodegeneration. Taken together, these data support a combined role of Gal-3 in Hp-mediated neuroin- flammation and neurodegeneration. Overexpression of Gal-3 by gastric mucosa macrophages in the context of $\mathrm{Hp}-\mathrm{I}$ may pro- vide molecular targets of structural similarity between epitopes expressed in the periphery, the BBB and the CNS, thus possibly triggering autoimmunity, neuroinflammation and, subsequently, neurodegeneration. We and others reported evidence of $\mathrm{Hp}-\mathrm{I}$ association with $C D$, either in the context of liver cirrhosis and/or primary neurodegen- erative disease. We previously also reported an association of $\mathrm{Hp}-\mathrm{I}$ with $\mathrm{AD}$, mild cognitive impairment [8], MS or MS-related clini- cally isolated syndrome (CIS), as well as a favorable effect of $\mathrm{Hp}$ eradication towards increasing AD survival and delaying CIS pro- gression into definite MS. Hp-I is common in cirrhotic patients with $\mathrm{HE}$ and it appears to be a frequent denominator connected with CD-related falls and fractures, as well as with post-HE persistent CD in patients with liver cirrhosis [8]. Hepatitis $C$ cirrhotic patients with detectable interferon- $\square$ and higher levels of IL-6 and TNF- $\square$ might be at risk of poor cognitive function. Hp-I may cause CD in the context of HE by: inducing hyperammonemia; promoting the release of proinflammatory and vasoactive sub- stances that further disrupt BBB; promoting platelet-leukocyte aggregation; and producing reactive oxygen metabolites or influ- encing 
apoptotic cellular processes [9]. Moreover, human defensins may contribute to Hp-related brain pathophysiology by modulating innate and adaptive immune responses. Specifically, human $\square$ - defensin-1 is upregulated in the context of $\mathrm{Hp}-\mathrm{I}$ and may serve as a biomarker of bacterial translocation in cirrhotic patients, thus signi- fying Hp-related extragastric complications, such as $\mathrm{CD}$ and $\mathrm{HE}$ [10]. Of note, similarly to defensins, Gal-3 also displays antimicrobial activity [2]. In view of the aforementioned data, we hereby suggest that Hp-related Gal-3 connected with MetS may play a role in the pathophysiology of cirrhosis-related chronic kidney, cardiovascu- lar and brain disorders and, thus, further studies are warranted to elucidate this field, which may have clinical and therapeutic implications. Conflict of interest None declared.

\section{References}

[1] Oikonomou T, Goulis I, Ntogramatzi F, et al. Galectin-3 is associated with glomerular filtration rate and outcome in patients with stable decompensated cirrhosis. Dig Liver Dis 2019.

[2] Sciacchitano S, Lavra L, Morgante A, et al. Galectin-3: One Molecule for an Alphabet of Diseases, from A to Z. Int J Mol Sci 2018;19.

[3] Boziki M, Polyzos SA, Deretzi G, et al. A potential impact of Helicobacter pylori-related galectin-3 in neurodegeneration. Neurochem Int 2018;113:13751. 
[4] Polyzos SA, Kountouras J. Helicobacter pylori infection and nonalcoholic fatty liver disease: Time for large clinical trials evaluating eradication therapy. Helicobacter 2019;24:e12588.

[5] Kountouras J, Polyzos SA, Zeglinas C, et al. Helicobacter pylori-related chronic hepatitis $\mathrm{C}$ infection and the risk for cardiovascular disease. Liver Int $2017 ; 37: 1082$.

[6] Wijarnpreecha K, Thongprayoon C, Nissaisorakarn P, et al. Association between Helicobacter pylori and end-stage renal disease: A meta-analysis. World J Gastroenterol 2017;23:1497-506.

[7] Kountouras J, Polyzos SA, Katsinelos P, et al. Cardio-cerebrovascular disease and Helicobacter pylori-related metabolic syndrome: We consider eradication therapy as a potential cardio-cerebrovascular prevention strategy. Int J Cardiol 2017;229:17-8.

[8] Kountouras J, Deretzi G, Zavos C, et al. Helicobacter pylori infection and liver cirrhosis: possible association with hepatic encephalopathy and/or posthepatic encephalopathy cognitive impairment in patients with portal hypertension. Ann Gastroenterol 2014;27:285.

[9] Kountouras J, Polyzos SA, Katsinelos P, et al. Potential Impact of Helicobacter Pylori on Hepatic Encephalopathy Pathophysiology. Dig Dis Sci 2018;63:1087-8.

[10] Kaltsa G, Bamias G, Siakavellas SI, et al. Systemic levels of human betadefensin 1 are elevated in patients with cirrhosis. Ann Gastroenterol 2016;29:63-70. 
